\title{
Do electrospray mass spectra of surfactants mirror their aggregation state in solution?
}

\author{
D. Bongiorno, ${ }^{a, b}$ L. Ceraulo, ${ }^{a, b}$ G. Giorgi, ${ }^{c}$ S. Indelicato ${ }^{a, b}$ and \\ V. Turco Liveri ${ }^{d *}$
}

\begin{abstract}
One important feature in the gas phase chemistry of surfactants is to ascertain whether their aggregates produced by electrospray ionization reflect those formed in the starting solution. With this aim, we have performed ESI-MS, ESI-MS/MS and ER-MS spectra of bis(2-ethylhexyl)sulfosuccinate (AOTNa) solutions in different solvents, i.e. water, water/methanol, methanol and $n$-hexane. The results clearly indicate that, notwithstanding the strongly different aggregation state in solution (direct micelles in water and in water/methanol, molecular dispersion in methanol and reverse micelles in $n$-hexane) and marked effects of the solvent polarity on the total ionic current, the surfactant aggregates in gas phase show identical structural features. Analogous conclusions can be drawn analyzing the infrared multiple photon dissociation (IRMPD) spectra of AOTNa solutions in water/methanol and $n$-hexane. Moreover, according to the idea that gas phase can be considered an apolar environment par excellence, data consistently suggest a reverse micelle-like aggregation. Some peculiarities of the mechanisms leading to aggregate formation through electrospray ionization of surfactant solutions in solvent media with different polarity have been also discussed. Copyright $\odot 2011$ John Wiley \& Sons, Ltd.
\end{abstract}

Keywords: AOT; surfactant; aggregation; IRMPD; mass spectrometry

\section{INTRODUCTION}

Recently, a steeply increasing interest has been devoted to the study of surfactant self-assembling in gas phase. This interest is certainly fuelled by the potential applications of these organized nanostructures but also by the intriguing questions posed by this subject. In particular, the structural organization of surfactant molecules within the gas phase aggregates, and if it depends on the surfactant nature and state in the starting solution, are two topics currently under debate.

By collecting ESI-MS spectra of several surfactants in aqueous solutions, Nohara et al. observed the occurrence of species with an average weight formally corresponding to that of the aggregates in solution. ${ }^{[1-4]}$ Following this idea, a method for the determination of the critical micelle concentration (CMC) of sodium bis(2-ethylhexyl) sulfosuccinate (AOTNa) in carbon tetrachloride by collecting TOF-SIMS spectra has been described. ${ }^{[5]}$ However, in contrast with this latter report and consistently with the intense solvent evaporation involved in electrospray ionization, we have observed the occurrence of surfactant aggregates using starting solutions below the CMC of AOTNa and independently either on the ionization technique (ESI-MS and MALDI-MS) or the polarity of the solvent used (water/methanol mixture or $n$-hexane). ${ }^{[6]}$

Concerning the surfactant structural organization in gas phase, it has been claimed that micellar structures in the starting solution can be preserved in the high vacuum conditions of the mass spectrometer and can survive the phase transition, without changing the orientation of the surfactant molecules. Hence, the charged surfactant aggregates observed in the gas phase should be considered as a picture of those present in the solution phase. ${ }^{[7,8]}$ However, while the residence time of aggregates within the mass spectrometer is of the order of milliseconds, recent molecular dynamics simulations showed that the characteristic rearrangement time taken by a nanodroplet of water surrounding a surfactant aggregate to revert in a watercontaining reverse micelle is about $10 \mathrm{~ns} .^{[9]}$

From an experimental point of view, Y. Fang et al. argued that AOTNa forms reverse micelle-like aggregates from their ability to encapsulate some small size amino acids in gas phase. ${ }^{[10,11]}$ Moreover, it has been recently reported that the charged aggregates obtained by electrospray ionization of sodium methane sulfonate, sodium butane sulfonate and sodium octane sulfonate in water-methanol solutions, studied by ESI and energy resolved mass spectrometry (ER-MS) and density functional theory calculations, are characterized by reverse micelle-like supramolecular organization. ${ }^{[12]}$ This suggests that, independent on their nature, surfactant molecules strongly prefer to form, in gas phase, reverse aggregates. Further support for a reverse micelle-like aggregation of surfactants in gas phase has been gained by

\footnotetext{
* Correspondence to: V. Turco Liveri, Dipartimento di Chimica "S. Cannizzaro", Università degli Studi di Palermo, Viale delle Scienze, 1-90128 Palermo, Italy. E-mail: turco@unipa.it

a Dipartimento di Scienze e Tecnologie Molecolari e Biomolecolari (STEMBIO), Università degli Studi di Palermo, Viale delle Scienze, Ed. 16, I-90128 Palermo, Italy

b Centro Grandi Apparecchiature-UniNetLab, Università degli Studi di Palermo, Via F. Marini 14, I-90128 Palermo, Italy

c Dipartimento di Chimica, Università degli Studi di Siena, Via Aldo Moro, I-53100 Siena, Italy

d Dipartimento di Chimica "S. Cannizzaro", Università degli Studi di Palermo, Viale delle Scienze, I-90128 Palermo, Italy
} 
collecting infrared multiple photon dissociation spectra of singly or double charged AOTNa aggregates formed in ESI experiments ${ }^{[13]}$ and by quantum chemical calculations and molecular dynamics simulation. ${ }^{[6,14]}$

Aiming to gain further insight into AOTNa self-assembling process and to understand if gas phase charged supramolecular aggregates are or not a mirror of the aggregation state in the starting solution, we carried out ESI-MS, ESI-MS/MS and energy resolved (ER-MS) experiments on AOTNa solutions in water, water/methanol (1:1 vol), methanol and $n$-hexane. With a similar purpose, IRMPD spectra on AOTNa solutions in water/methanol (1:1 vol) and in pure $n$-hexane were also collected.

AOTNa has been chosen owing to its peculiar ability to form direct micelles when dissolved in polar media and reverse micelles in apolar environments. ${ }^{[15-18]}$ In particular, it must be stressed that AOTNa forms in water (dielectric constant $(\varepsilon)=78$ ) and in 1:1 water-methanol mixture $(\varepsilon=59)$ direct micelles above the system specific critical micellar concentration (CMC). The CMC in water is in the range 2.5-6 $10^{-3} \mathrm{M}^{[19]}$ whereas a higher $C M C$ value is expected for the 1:1 water/methanol mixture. ${ }^{[19-21]}$ On the other hand, AOTNa is molecularly dispersed in methanol $(\varepsilon=33)$ whereas it forms reverse micelles in $\mathrm{n}$-hexane $(\varepsilon=1.9)$ above a CMC value of about $10^{-4} \mathrm{M}^{[19,22]}$

\section{EXPERIMENTAL SECTION}

Sodium bis-(2-ethylhexyl) sulfosuccinate (AOTNa, 99\%) was purchased from Sigma-Aldrich (Germany). Solutions were prepared using water, methanol and $n$-hexane of LC grade.

Q-ToF experiments were carried out using a Q-ToF Premier mass spectrometer (Waters, Manchester, UK) equipped with an electrospray source (ESI).

The ESI-MS experiments were performed in positive ion mode using AOTNa solutions at fixed surfactant concentration $(0.5 \mathrm{mM})$ in various solvent media with different polarity, i.e. water, water/ methanol (1:1), methanol and in $n$-hexane at $\mathrm{W}=10(\mathrm{~W}=$ [water]/ [AOTNa]). It must be stressed that the addition of water to the latter solution was necessary in order to permit the formation of charged species. In fact, no charged species were observed in anhydrous AOTNa/n-hexane samples.

The solutions were infused at $5 \mu \mathrm{min}^{-1}$ flow rate under a spray voltage of $3.5 \mathrm{kV}\left(50 \mu \mathrm{lmin}^{-1}\right.$ and $5 \mathrm{kV}$ in the case of the water/AOTNa/n-hexane sample). The sampling cone was set at $170 \mathrm{~V}$, and the CID gas was Argon. The other operative conditions were: extraction cone $3.2 \mathrm{~V}$, ion guide $1.2 \mathrm{~V}$, source temperature $90^{\circ} \mathrm{C}$, desolvation temperature $250{ }^{\circ} \mathrm{C}$, desolvation gas $530 \mathrm{I} \mathrm{h}^{-1}$, cone gas flow $50 \mathrm{lh}^{-1}$, RF settings autoprofile, mass range $50-4000 \mathrm{~m} / \mathrm{z}$, scan time $1 \mathrm{~s}$.

Since the experimental conditions used for the water/AOTNa/ $n$-hexane samples are different from those of AOTNa in the other solvent media, the comparison with the results of the latter samples will be only qualitative.

MS/MS spectra were recorded under following experimental conditions: spray voltage $3.5 \mathrm{kV}$ ( $5 \mathrm{kV}$ for water/AOTNa/n-hexane solutions), sampling cone $170 \mathrm{~V}$, extraction cone $3.2 \mathrm{~V}$, ion guide $1.2 \mathrm{~V}$, source temperature $90^{\circ} \mathrm{C}$, desolvation temperature $250^{\circ} \mathrm{C}$, desolvation gas $50 \mathrm{I} \mathrm{h}^{-1}$, collision gas argon, scan time $1 \mathrm{~s}$.

The survival yield (SY) curves of AOTNa aggregates at $\mathrm{m} / \mathrm{z} 1355$ and 2687 were obtained using the same experimental procedure used for ESI-MS/MS experiments, except the collision energies that were changed by discrete values ranging from 5 to $100 \mathrm{~V}$ during the flow injection experiments. For each collision energy (CE) value, an acquisition time of 1 min was set. Each ESI-MS/ MS spectrum was obtained by the average of 11 consecutive scans. The percentage of each fragment was calculated dividing the counts of the correspondent peak to the sum of counts of all peaks including the precursor ion.

Experimental conditions were maintained constant through the complete set of measurements, and each SY curve of positively monocharged aggregates was repeated three times. All analyses were recorded in the same experimental section and in the same day. Moreover, to avoid possible instrumental errors, SY curves were recorded alternatively using polar and apolar solutions.

The SY and $Y_{i}$ reported in both text and figures are defined according to the Eqns (1) and (2), respectively:

$$
\begin{gathered}
S Y=\frac{I_{M}}{I_{M}+\sum I_{F}} \\
Y_{i}=\frac{I_{F, i}}{I_{M}+\sum I_{F}}
\end{gathered}
$$

Where $I_{M}$ and $I_{F, i}$ are the intensities of the ionized molecule and of the fragment $i$, respectively, and $\sum I_{F}$ is the sum of all fragment ions intensities.

\section{IRMPD measurements}

Measurements were carried out at the FELIX facility (FOM Rijnhuizen, Netherlands). The experimental apparatus consists in a Fourier transform ion cyclotron mass spectrometer equipped with an electrospray ion source (Micromass Z-spray) and coupled to the infrared laser beam of FELIX. The high power in a wide frequency range of FELIX makes it an ideal probe of the structure of supramolecular aggregates in gas phase. ${ }^{[23]}$

A $2.7 \times 10^{-3} \mathrm{M}$ solution of AOTNa in water/methanol (50/50 \% vol) or $8.2 \times 10^{-3} \mathrm{M}$ solution of AOTNa in $n$-hexane at $W=10$ $(\mathrm{W}=[$ water $] /[\mathrm{AOTNa}])$ has been introduced via direct infusion at a flow rate of $10 \mu \mathrm{L} \mathrm{min} \mathrm{m}^{-1}$. At each wavenumber $(v)$ of the exciting infrared laser radiation, three mass spectra were acquired, and the averaged integrated intensities of the parent ion $\left(I_{p}(v)\right)$ and of its fragments $\left(I_{f, i}(v)\right)$ are employed to calculate the fragmentation degree $Y(v)$ according to Eqn (3):

$$
Y(v)=\frac{\sum_{i} I_{f, i}(v)}{I_{p}(v)+\sum_{i} I_{f, i}(v)}
$$

\section{RESULTS AND DISCUSSIONS}

It is well known that, driven by the hydrophobic effect and above a critical concentration (CMC), surfactant molecules form direct micelles in aqueous solutions, thus avoiding contacts between the surfactant alkyl chains and the surrounding water molecules. In such globular aggregates, surfactant molecules are oriented so that the alkyl chains form the internal core, while hydrated head groups constitute the external layer. Another interesting feature of surfactant aqueous solutions is the formation at the water/air interface of a layer of surfactant molecules oriented so that the polar head groups are dipped in the liquid phase while the alkyl chains protrude toward the gas phase. In this contest, it is worth 
to note the central role of polar solvent molecules to force these surfactant self-assembling modes as well as the existence of a surfactant-oriented monolayer in the charged nanodroplets produced by electrospray ionization. Then, through intense solvent evaporation (involving local increase of surfactant concentration), Coulomb explosions (leading to the formation of smaller sized droplets), ejection of charged species ions from highly charged droplet surface, the observed species are produced.

On the other hand, the formation of reverse micelles in apolar media is the consequence of favorable interactions between the surfactant hydrophilic heads, while the solvent plays the minor role of dispersing medium. This involves the building up of aggregates characterized by an internal core consisting of polar moieties surrounded by an apolar layer formed by the surfactant alkyl chains. Obviously, no enrichment of surfactant molecules at the apolar medium/air interface should be expected. ${ }^{[15-18]}$

However, the formation of a highly charged layer in proximity of the surface of apolar nanodroplets produced by electrospray ionization should affect the surface molecular composition. Thus, also in the case of surfactant solutions in apolar solvents, it can be expected that the observed charged species arise from solvent evaporation, Coulomb explosions and ejection of charged species ions from droplet surface. However, since these processes are system specific, significant departures from the electrospray of surfactant solutions in polar media can be predicted.

Taking into account that vacuum can be considered an apolar environment par excellence, it can be expected that in such environment, surfactant molecules prefer a reverse micelle-like aggregation mode. However, since electrospray ionization produces charged aggregates in strongly out-of-equilibrium conditions, it could be suspected that these aggregates should reflect both their state in solution and the specific conditions under which electrospray ionization is carried out.

To answer these questions, here, we will discuss the results of our ESI-MS, ESI-MS/MS, ER-MS and IRMPD experiments on AOTNa solutions in water, water/methanol (1:1 vol), methanol and $n$-hexane at $W=10(\mathrm{~W}=$ [water]/[AOTNa]).

Typical ESI-MS spectra in positive ion mode of the AOTNa in water, water/methanol, methanol and $n$-hexane solutions are shown in Fig. 1a-d. It is worth to note that all the spectra show the same peaks due to the monocharged aggregates up to $\mathrm{m} / \mathrm{z} 3575$ corresponding to the $\left[\mathrm{AOT}_{n} \mathrm{Na}_{\mathrm{n}+1}\right]^{+}$species with $n$ from 1 to 8 together with some peaks at lower $m / z$ values arising from AOTNa fragmentation. ${ }^{[6]}$ Moreover, the peaks of singly charged monomer and trimer species, at $m / z 467$ and 1355, respectively, dominate in all these spectra. ${ }^{[6,12]}$

On the other hand, the different abundance distributions are attributable more likely to the different nature of solvents. In addition, a progressive increase of the total ionic current (TIC) is observed by increasing the solvent medium dielectric constant, due to the enhanced stabilization of charge separation in solution which, in turn, makes more easy the formation of charged species under electrospray ionization conditions. ${ }^{[24]}$

In particular, as shown in Fig. 2 where the results collected in the same experimental conditions are reported, a nearly linear trend of TIC as a function of the dielectric constant is observed. Incidentally, the behavior of AOTNa solution in water, which is somewhat at odds with the notion that aqueous solutions are not suitable for electrospray, emphasizes the huge decrease of the surface tension due to the surfactant monolayer at the droplet surface.

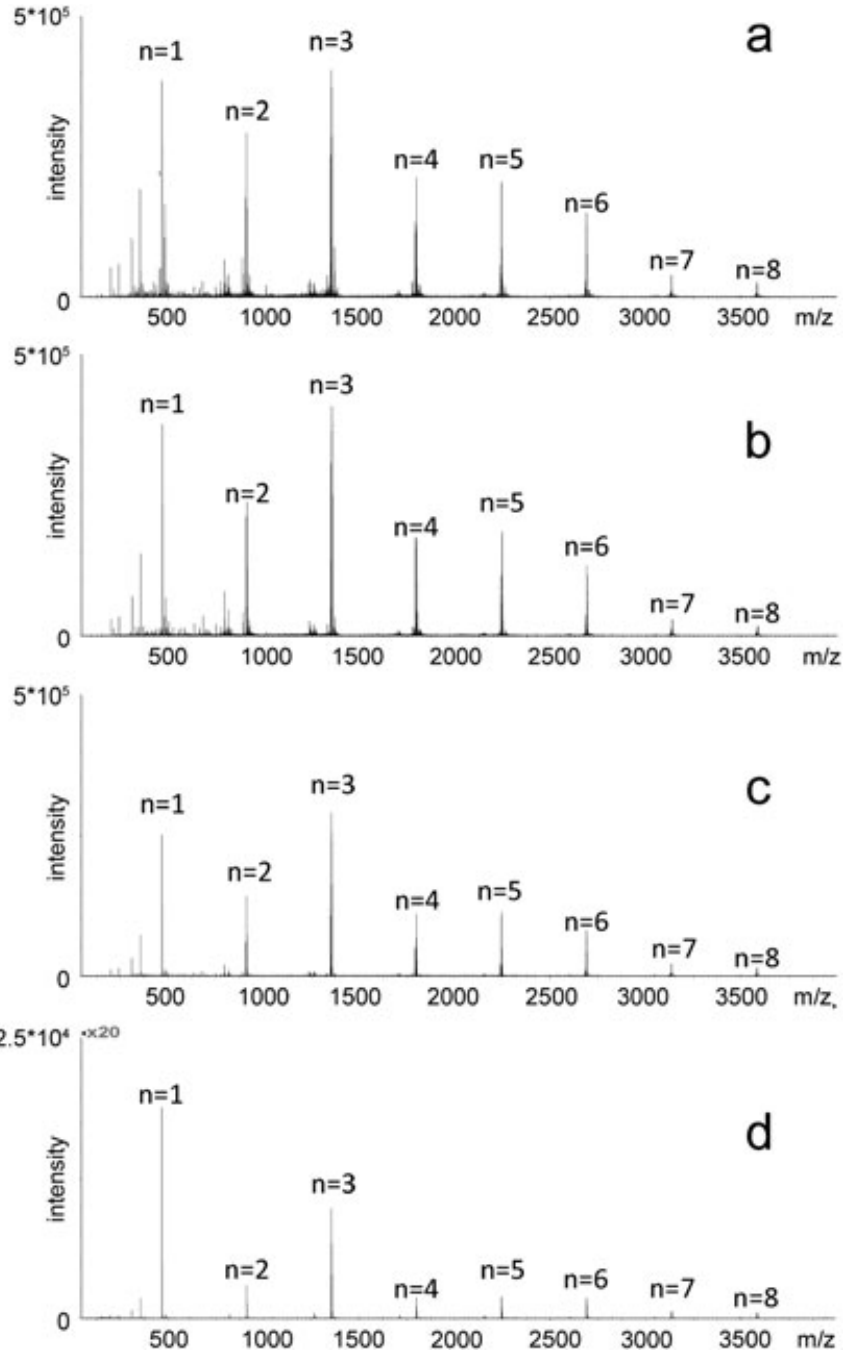

Figure 1. ESI-MS full scan spectra of AOTNa solutions in a: water, b: water/ methanol (1:1), c: methanol and $\mathbf{d}$ : $n$-hexane. The top labels on the peaks refer to the aggregation number of $\left[\mathrm{AOT}_{n} \mathrm{Na}_{n+1}\right]^{+}$species. In the $\mathrm{Y}$ axis is reported the absolute intensity expressed as counts.

Besides, Fig. $2 \mathrm{~b}$ shows that the increase of the medium dielectric constant affects differently the abundance of the various aggregates favoring the more abundant ones.

In order to establish if AOTNa aggregates, obtained by the various starting solutions, have the same structural arrangement and if their relative stabilities depend or not on polarity of ESI solvent, ER-MS measurements have been carried out. To this aim, we have selected the $\left[\mathrm{AOT}_{3} \mathrm{Na}_{4}\right]^{+}$and $\left[\mathrm{AOT}_{6} \mathrm{Na}_{7}\right]^{+}$as representative aggregates. Indeed, a previous work ${ }^{[13]}$ reported that the trimer and bigger aggregates have practically superimposable IRMPD spectra, supporting a very close structural similarity and strongly suggesting the same aggregation pattern. The $\mathrm{MS} / \mathrm{MS}$ spectra for $\left[\mathrm{AOT}_{3} \mathrm{Na}_{4}\right]^{+}$and $\left[\mathrm{AOT}_{6} \mathrm{Na}_{7}\right]^{+}$aggregates, under the experimental conditions previously reported, show the loss of neutral $\left[(\mathrm{AOTNa})_{n}\right]$ species $^{[25]}$ as main decomposition pathways and are practically identical irrespective of the nature of the solvent in the starting solution.

Even if these data strongly suggest that the structures of $\left[\mathrm{AOT}_{3} \mathrm{Na}_{4}\right]^{+}$and of $\left[\mathrm{AOT}_{6} \mathrm{Na}_{7}\right]^{+}$are independent on the ESI solvent, it seemed useful also to verify their SY and the yield $(Y)$ 

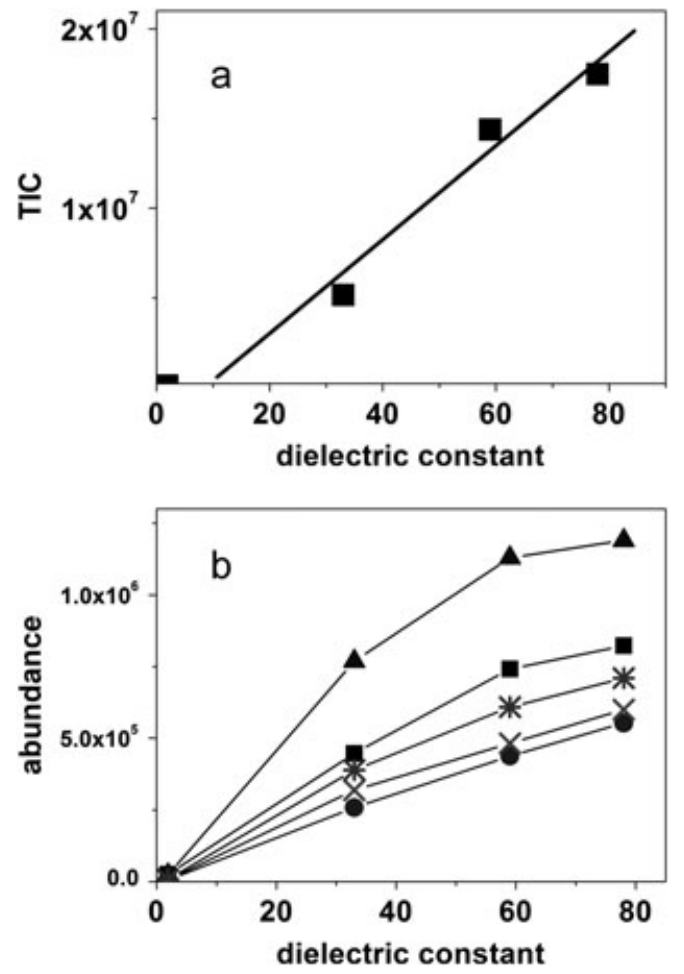

Figure 2. a: TIC value as a function of the solvent dielectric constant; b: Abundance of some representative species: $[\mathrm{AOT} \mathrm{Na}]^{+}, \mathbf{m} ;\left[\mathrm{AOT}_{2} \mathrm{Na}_{3}\right]^{+}, \mathbf{\bullet}$; $\left[\mathrm{AOT}_{3} \mathrm{Na}_{4}\right]^{+}, \mathbf{\Delta} ;\left[\mathrm{AOT}_{4} \mathrm{Na}_{5}\right]^{+}, \mathrm{X} ;\left[\mathrm{AOT}_{5} \mathrm{Na}_{6}\right]^{+}$, ; as a function of the solvent dielectric constant.

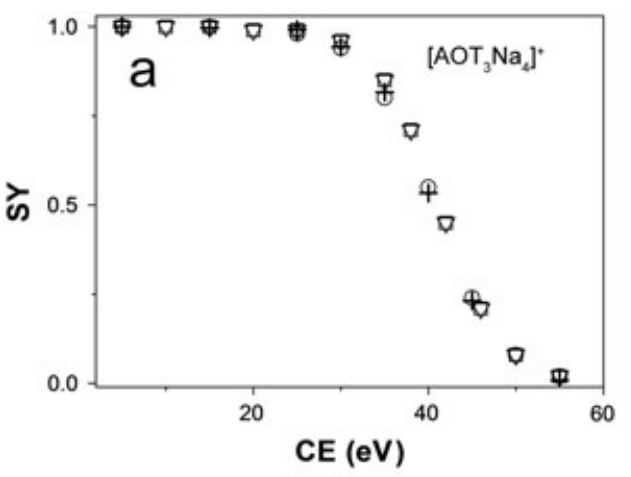

curves of the parent and fragment ions as function of the laboratory CE (breakdown curves). ${ }^{[26-28]}$

The SY values versus the $\mathrm{CE}$ applied of $\left[\mathrm{AOT}_{3} \mathrm{Na}_{4}\right]^{+}$and $\left[\mathrm{AOT}_{6} \mathrm{Na}_{7}\right]^{+}$obtained from all the starting solutions are shown in Figs. $3 a$ and $3 c$, whereas Figs. $3 b$ and $3 d$ show the yield of the fragment ions as a function of the CE.

It is worth noting that the SY curves for 3-mer and 6-mer species as well as the $Y$ of the fragments are practically overlapped. This means that, independent on the ESI solvent, both $\left[\mathrm{AOT}_{3} \mathrm{Na}_{4}\right]^{+}$and $\left[\mathrm{AOT}_{6} \mathrm{Na}_{7}\right]^{+}$undergo identical collision-induced fragmentations also by a quantitative point of view in a wide range of collision energies. Taking into account that direct and reverse aggregates should be characterized by very different stabilization energy, this result indicates that the same aggregate is observed, independent of the surfactant aggregation state in the starting solution and of the effect of the solvent polarity on the electrospray ionization process. Incidentally, it is worth to note that ER-MS, generally used to gain information on the stability of a selected ion, is here used as a valuable tool for supramolecular structure elucidation.

Additional support to the above reported findings was gained by IRMPD spectra of the $\left[\mathrm{AOT}_{3} \mathrm{Na}_{4}\right]^{+}$species obtained by ESI from infusion of the AOTNa solutions in $n$-hexane and in water/ methanol. These spectra are shown in Fig. 4. According to literature, all the observed bands are due to functional group of AOTNa. ${ }^{[29,30]}$ Of particular interest is the sharp peak at $1722 \mathrm{~cm}^{-1}$ due to the AOTNa CO stretching and that at $1051 \mathrm{~cm}^{-1}$ due to the symmetric stretching of the AOTNa $\mathrm{SO}_{3}^{-}$group because the positions of both these peaks are sensitive probe of their local environments and consequently of the aggregation state of AOTNa molecules. ${ }^{[13]}$
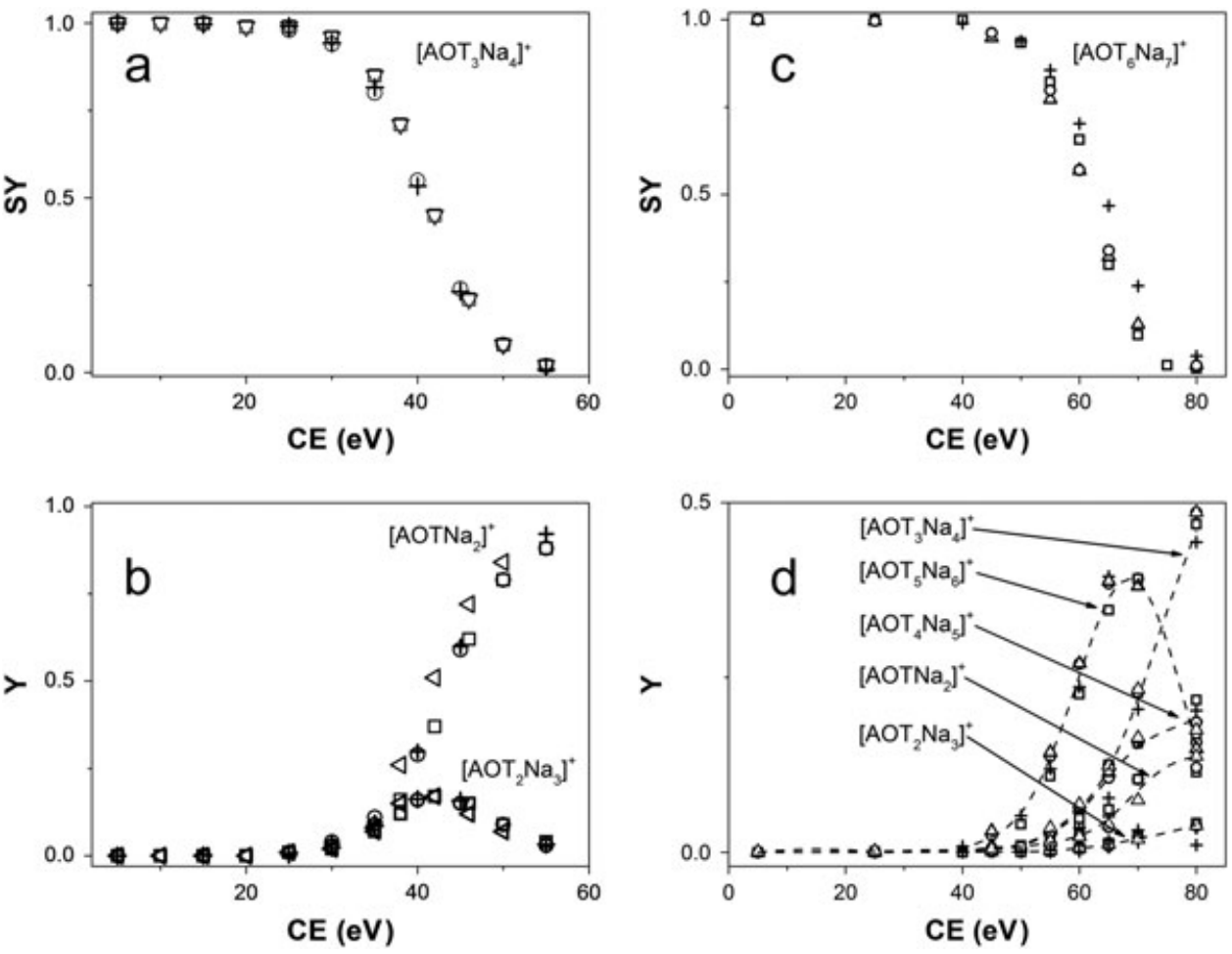

Figure 3. a and c: SY curves for $\left[\mathrm{AOT}_{3} \mathrm{Na}_{4}\right]^{+}$and $\left[\mathrm{AOT}_{6} \mathrm{Na}_{7}\right]^{+}$, respectively, $\mathbf{b}: Y$ curves of $\left[\mathrm{AOT} \mathrm{Na}_{2}\right]^{+}$and $\left[\mathrm{AOT}_{2} \mathrm{Na}_{3}\right]^{+}$arising from the fragmentation of $\left[\mathrm{AOT}_{3} \mathrm{Na}_{4}\right]^{+}, \mathbf{d}: Y$ curves of $\left[\mathrm{AOT}_{n} \mathrm{Na}_{n+1}\right]^{+}$fragments arising from the fragmentation of $\left[\mathrm{AOT}_{6} \mathrm{Na}_{7}\right]^{+}$, obtained by AOTNa ESI MS/MS experiments performed in various solvent media as a function of CE. Solvents used: water, $\square$; water/methanol, ; methanol, $O$; $n$-hexane, + . Dashed lines are reported as guide for the eyes. 


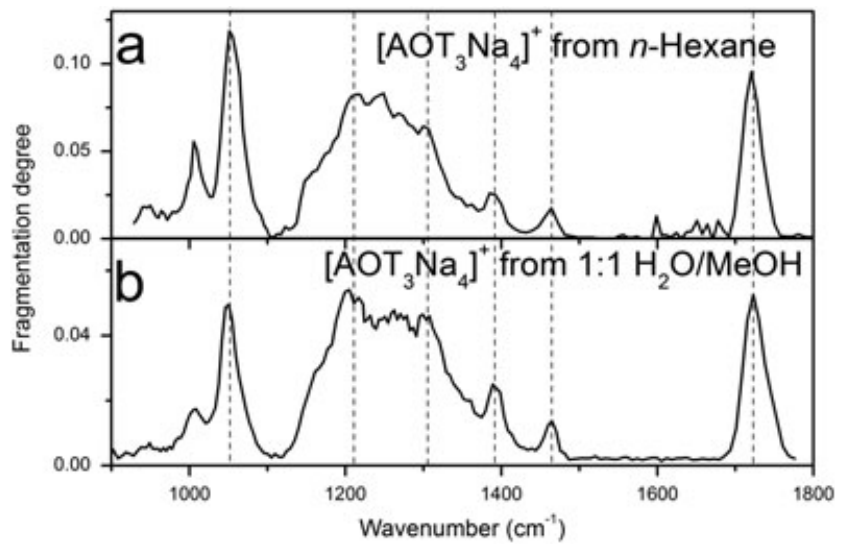

Figure 4. IRMPD spectra of AOTNa positively monocharged trimers obtained by infusion of surfactant solution in $\mathbf{a}$ : hexane and $\mathbf{b}$ : water/ methanol.

The comparison of the spectra indicates that no significant change is observed meaning that no memory effect of the surfactant state in the starting solution occurs.

Moreover, the positions of the AOTNa $\mathrm{CO}$ and $\mathrm{SO}_{3}^{-}$stretchings indicate that these groups are arranged so that they are in close contact among them and with the sodium counterions, a picture which is consistent with a reverse micelle-like aggregation. ${ }^{[13]}$

\section{CONCLUSIONS}

Crucial experiments to answer the vexed point if gas phase surfactant aggregates mirror their aggregation in solution have been carried out by performing ESI-MS, ESI-MS/MS, ER-MS and IRMPD measurements. We have chosen sodium bis(2-ethylhexyl)sulfosuccinate as paradigmatic surfactant, to take advantage of its ability to form both direct micelles in polar media and reverse micelles in apolar ones.

It was found that, notwithstanding the different aggregation state in solution, the surfactant aggregates in gas phase present identical structural features. Considering that electrospray ionization produces out-of-equilibrium surfactant aggregates, our experimental findings suggest that a rapid rearrangement of aggregates during the electrospray ionization stage and before entering the collision cell should take place. This poses a superior limit to the aggregate rearrangement of about $1 \mathrm{~ms}$, which is consistent with recent molecular dynamics calculation of the rearrangement time of about $10 \mathrm{~ns}^{[9]}$ Thus, our experimental findings together with theoretical calculations allow to claim that ESI spectra do not mirror the aggregation state of surfactants in solution.

On the other hand, the change of solvent polarity affects significantly the total ion current and consequently the abundance of the various aggregates favoring preferentially the most abundant ones. Finally, according to the idea that vacuum can be considered an apolar environment par excellence, data consistently suggest a reverse micelle-like aggregation.

\section{Acknowledgments}

The support of University of Palermo (Fondi di Ricerca Scientifica ex $60 \%$ ) is gratefully acknowledged. Mass spectrometry experimental data were provided by Centro Grandi Apparecchiature, UniNetLab, Università di Palermo funded by P.O.R. Sicilia
2000-2006, Misura 3.15 Quota Regionale. Further funding has been received from the European Community's Seventh Framework Programme (FP7/2007-2013) under Grant Agreement No. 226716. The excellent support by the FELIX staff is gratefully acknowledged.

\section{REFERENCES}

[1] D. Nohara, T. Tohkoshi, T. Sakai. The Possibility of the Direct Measurement of Micelle Weight by Electrospray Ionization Mass Spectrometry. Rapid Commun. Mass Spectrom. 1998, 12, 1933.

[2] D. Nohara, M. Bitoh. Observation of micelle solution of decyltrimethylammonium bromide by electrospray ionization mass spectrometry. J. Mass Spectrom. 2000, 35, 1434.

[3] D. Nohara, M. Bitoh. Measurement and analysis of ESI mass spectra of micelle solution. Adv. Mass Spectrom. 2001, 15, 801.

[4] D. Nohara, T. Kajiura, K. Takeda. Determination of micelle mass by electrospray ionization mass spectrometry. J. Mass Spectrom. 2005, $40,489$.

[5] S. A. Burns, P. L. Valint, J. A. Gardella. Determination of Critical Micelle Concentration of Aerosol-OT Using Time- of-Flight Secondary Ion Mass Spectrometry Fragmentation Ion Patterns. Langmuir 2009, 25, 11244.

[6] D. Bongiorno, L. Ceraulo, A. Ruggirello, V. Turco Liveri, E. Basso, R. Seraglia, P. Traldi. Surfactant Self-Assembling in Gas Phase: Electrospray lonization and Matrix-Assisted Laser Desorption/lonization-Mass Spectrometry of Singly Charged AOT Cluster. J. Mass Spectrom. 2005, 40, 1618.

[7] M. Sharon, L. L. llag, C. V. Robinson. Evidence for micellar structure in the gas phase. J. Am. Chem. Soc. 2007, 129, 8740.

[8] C. L. Hanson, L. L. Ilag, J. Malo, D. M. Hatters, G. J. Howlett, C. V. Robinson. Phospholipid complexation and association with apolipoprotein C-II - Insights from Mass Spectrometry. Biophys. J. 2003 $85,3802$.

[9] D. van der Spoel, E. G. Marklund, D. S. D. Larsson, C. Caleman Proteins, Lipids, and Water in the Gas Phase. Macromol. Biosci. 2011, 11, 50 .

[10] Y. Fang, A. Bennett, J. Liu. Multiply charged gas-phase NaAOT reverse micelles: Formation, encapsulation of glycine, and collisioninduced dissociation. Int. J. Mass Spectrom. 2010, 293, 12.

[11] Y. Fang, A. Bennett, J. Liu. Selective transport of amino acids into the gas phase: driving forces for amino acid solubilization in gas-phase reverse micelles. Phys. Chem. Chem. Phys. 2011, 13, 1466.

[12] D. Bongiorno, L. Ceraulo, G. Giorgi, S. Indelicato, M. Ferrugia, A. Ruggirello, V. Turco Liveri. Effects of the net charge on abundance and stability of supramolecular surfactant aggregates in gas phase. J. Mass Spectrom. 2011, 46, 195.

[13] G. Giorgi, L. Ceraulo, G. Berden, J. Oomens, V. Turco Liveri. Gas phase infrared multiple photon dissociation spectra of organized positively charged sodium bis-(2-ethylhexyl)sulfosuccinate reverse micelle like aggregates. J. Phys. Chem. B 2011, 115, 2282.

[14] G. Longhi, S. L. Fornili, V. Turco Liveri, S. Abbate, D. Rebeccani, L. Ceraulo, F. Gangemi. Sodium bis(2-ethylhexyl)sulfosuccinate selfaggregation in vacuo: molecular dynamics simulation. Phys. Chem. Chem. Phys. 2010, 12, 4694.

[15] H. F. Eicke. Topics in Current Chemistry, Vol. 87, F. L. Bosche (Ed.). Springer-Verlag: New York, 1980, 85-145.

[16] P. L. Luisi, L. J. Magid. Solubilization of enzymes and nucleic acids in hydrocarbon micellar solutions. CRC Crit. Rev. Biochem. 1986, 20, 409.

[17] P. Stilbs, B. Lindman. Aerosol OT aggregation in water and hydrocarbon solution from NMR self- diffusion measurements. J. Colloid Interface Sci. 1984, 99, 290.

[18] E. Y. Sheu, S. H. Chen, J. S. Huang. Structure and Growth of Bis (2ethylhexyl) Sulfosuccinate Micelles in Aqueous Solutions. J. Phys. Chem. 1987, 91, 3306.

[19] F. Heatley. $\mathrm{A}^{1} \mathrm{H}^{13} \mathrm{C}$ nuclear magnetic resonance study of the conformation of aerosol OT in water and hydrocarbon solutions. J. Chem. Soc. Faraday Trans. I, 1987, 83, 517.

[20] P. S. Albright, L. J. Gosting. Dielectric constant of the methanol-water system from 5 to $55^{\circ} \mathrm{C}$. J. Am. Chem. Soc. 1946, 68, 1061.

[21] M. Ueno, H. Kishimoto, Y. Kyogoku. 13C-NMR Study of aerosol OT in aqueous and organic solvents. J. Coll. Int. Sci. 1978, 63, 113.

[22] M. J. Hossain, Y. Hayashi, N. Shimizu, T. Kawanishi. Determination of Critical Micelles Concentration of Aerosol OT/Isooctane Reverse 
Micelles by Fourier Transformed Infrared Spectroscopy (FTIR). J. Chem. Eng. Jpn. 1996, 29, 381.

[23] N. C. Polfer, J. J. Valle, D. T. Moore, J. Oomens, J. R. Eyler, B. Bendiak. Differentiation of Isomers by Wavelength-Tunable Infrared MultiplePhoton Dissociation-Mass Spectrometry: Application to GlucoseContaining Disaccharides. Anal. Chem. 2006, 78, 670.

[24] R. B. Cole. Some tenets pertaining to Electrospray ionization mass spectrometry. J. Mass Spectrom. 2000, 35, 763.

[25] D. Bongiorno, L. Ceraulo, G. Giorgi, S. Indelicato, A. Ruggirello, V. Turco Liveri. Supramolecular Aggregates In Vacuum: Positively Monocharged Sodium Alkanesulfonate Clusters. Eur. J. Mass Spectrom. 2010, 16, 151.

[26] F. Derwa, E. D. Pauw, P. Natalis. New basis for a method for the estimation fo seconary ion internal energy distribution in 'soft' ionization techniques. Org. Mass Spectrom. 1991, 26, 117.
[27] X. H. Guo, M. C. Duursma, P. G. Kistemaker, N. M. M. Nibbering, K. Vekey, L. Drahos, R. M. A. Heeren. Manipulating internal energy of protonated biomolecules in electrospray ionization Fourier transform ion cyclotron resonance mass spectrometry. J. Mass Spectrom. 2003, 38, 597.

[28] C. Collette, L. Drahos, E. D. Pauw, K. Vékey. Comparison of the internal energy distributions of ions produced by different electrospray sources. Rapid Commun. Mass Spectrom. 1998, 12, 1673.

[29] Q. Li, S. Weng, J. Wu, N. J. Zhou. Comparative Study on Structure of Solubilized Water in Reversed Micelles. 1. FT-IR Spectroscopic Evidence of Water/AOT/n-Heptane and Water/NaDEHP/n-Heptane Systems. J. Phys. Chem. B 1998, 102, 3168.

[30] P. D. Moran, G. A Bowmaker, R. P. Cooney, J. R. Bartlett, J. L. Woolfrey. Vibrational spectra of metal salts of bis(2-ethylhexyl)sulfosuccinate (AOT). J. Mater. Chem. 1995, 5, 295. 